\title{
Von Zwergen und Intellektuellen
}

Erhard Taverna

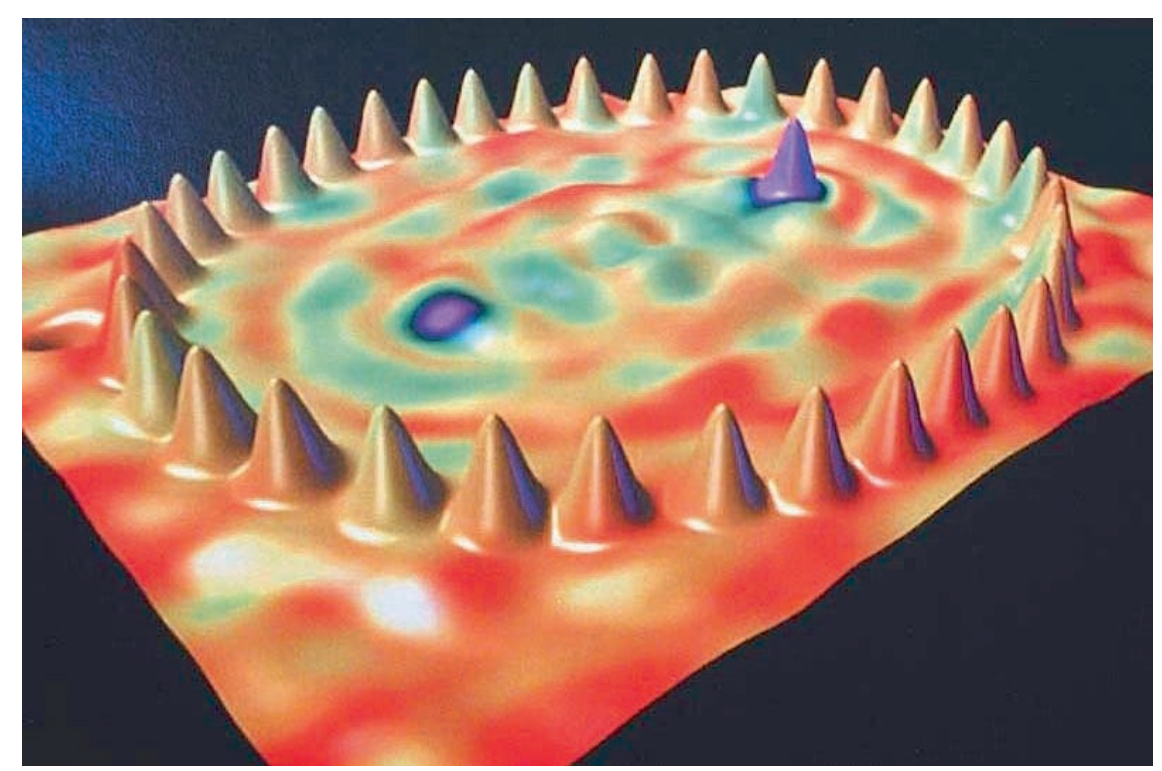

Wer soll uns in unübersichtlichen Zeiten mit einer mutigen Geste zu einer Perspektive verhelfen? Genügt es, die unterschiedlichsten Experten medienwirksam an einen Tisch zu holen und die Politiker mit teuren Gutachten einzudecken? Erschöpft sich der intellektuelle Diskurs in der Mediation arbeitsteilig reflektierender Akteure, vielstimmig und ohne Anspruch auf Kohärenz oder Nachhaltigkeit? Das Zentrum für Technologiefolgen-Abschätzung TA-SWISS untersucht im Auftrag des Bundes die Chancen und Risiken neuer technologischer Entwicklungen in den Bereichen Biotechnologie und Medizin, Informationsgesellschaft und Nanotechnologie. Auch die Fachzeitschrift «Oekoskop» der Ärztinnen und Ärzte für Umweltschutz thematisiert die letzterwähnte Schlüsseltechnologie: «Nanopartikel/ Nanotechnologie». Die Philosophin und Professorin Sabine Maasen von der Universität Basel analysiert mit ihrem Beitrag das Dilemma einer Institution der Politikberatung, zu deren Entwicklung sie massgeblich beigetragen hat.

TA-Studien beeinflussen mit ihren Empfehlungen und Resultaten den Fluss der öffentlichen Fördermittel und damit die Forschungsanstrengungen innerhalb und vor allem ausserhalb der Universitäten. Besonders in Abstimmungen kommt dabei ein Argument zum Tragen, das die Autorin als «Ökonomie des Versprechens» umschreibt. Ein typisches Beispiel war der Abstim- 
Bertolt Brechts Galilei betrachtet sich als Versager, denn es genüge nicht, nur Entdecker zu sein: «Die Bewegungen der Himmelskörper sind übersichtlicher geworden; immer noch unberechenbar sind den Völkern die Bewegungen der Herrscher.» Wissenschaft erfordere besondere Tapferkeit, denn mit dem Zweifel als Arbeitsprinzip müsse sie alle Menschen zu Zweiflern machen, damit diese sich vom «perlmutternen Dunst von Aberglauben und alten Wörtern» befreien können. Das einzige Ziel der Wissenschaft bestehe darin, die Mühseligkeit der menschlichen Existenz zu erleichtern. Doch resigniert stellt er fest: «Wie es nun steht, ist das Höchste, was man erhoffen kann, ein Geschlecht erfinderischer Zwerge, die für alles gemietet werden können.»

Genügt es den Konsumenten, wenn ihre Brillengläser besser verspiegelt und die lackierten Lieblingsspielzeuge schmutzabstossender werden? Leben wir nicht längst ein fragmentiertes Dasein im galileischen Zwergenstaat von Fachidioten, fremdbestimmt vom Wachstumszwang einer ökonomischen Lehre, die zum Selbstzweck degenerierte? Die Dynamik der «Wissensgesellschaft» ist trotz TA nicht mehr steuerbar, eine staatliche Planung, die der Wettbewerbslogik widerspricht, nicht mehr möglich. Was soll da ein universal denkender Intellektueller? Ist er der Antipode mit dem Durchblick, den wir bräuchten? Er oder sie müsste vielen Ansprüchen genügen, sollte ungefiltert und illusionslos beobachten, das Augenmass behalten, ein gelassenes Urteil fällen können, niemals korrupt, dafür klug, weise und schöpferisch sein und verantwortungsvoll an der erwarteten Synthese mitarbeiten. Gemäss dem Wissenschaftstheoretiker und Publizisten Holger Gumprecht hat der intellektuelle Fährtenleser neue Funktionen zu übernehmen: «Statt nur Fackelträger in der Nacht wären die neuen Intellektuellen Katalysatoren von Komplexität in einer stets von zuviel Struktur, von zuviel Organisation, von Negentropie (Übermass an Information) eher als von Orientierungslosigkeit bedrohten Kultur.»

- www.ta-swiss.ch/a/meth_doku/2006_TAD

- Geißel B, Penrose V. Lokale Vernetzung und Wissensintegration von Laien(-wissen) und Experten (-wissen) durch neue Partizipationsformen. www. sciencepolicystudies.de/dok/expertise-geissel.pdf.

- Oekoskop Nr. 4/07, Fachzeitschrift der Ärztinnen und Ärzte für Umweltschutz. www.aefu.ch

\section{Grundsätzliches und praktische Ratschläge zur Führung aus persönlicher Erfahrung}

\section{Richard O. Binswanger* \\ * Der Autor ist seit 28 Jahren Chefarzt in der Radiologie, seit 1995 am Kantonsspital Münsterlingen. Er hat sich in der ganzen Zeit intensiv mit Führung befasst und 2004 die Führungsschule Bodensee Mün- sterlingen gegründet. Dementspre- chend erfolgte auch eine intensive Beschäftigung mit theoretischen Führungsfragen. Es handelt sich um seine ganz persönliche Sicht.}

Korrespondenz:

Dr. med. Richard.O. Binswanger Chefarzt Radiologie Spital Thurgau AG Kantonsspital

Führungsschule

Bodensee Münsterlingen

CH-8596 Münsterlingen

r.binswanger@bluewin.ch

www.fsb-spital.ch

\section{Merksätze}

Als Führer sind Sie in der Pflicht

Sie sind der oberste Diener Ihrer Organisation.

Im Zentrum steht die Aufgabe

Sie muss formuliert und verstanden sein. Aus ihr entwickeln sich Struktur und Kultur.

\section{Bleiben Sie Sie selbst}

Entscheidend ist nicht Ihr Führungsstil, sondern dass Sie glaubhaft sind.

Verlangen Sie Leistung, Respekt und Loyalität Von sich selbst und Ihren Mitarbeitern.

Schenken Sie Vertrauen

Aber seien Sie wachsam.
Verlangen Sie Lösungsvorschläge, nicht Probleme I han e Lösig, nicht: i han es Problem.

Seien Sie ehrlich

Tut oft unheimlich weh, aber Lügen haben kurze Beine.

\section{Schärfen Sie die Urteilskraft}

Ihre eigene und die Ihrer Mitarbeiter.

Entscheiden Sie

Führen heisst, das Unentscheidbare zu entscheiden. Das ist schwer, und Fehlentscheide sind unausweichlich. Die meisten lassen sich korrigieren. Tun Sie dies so bald als möglich.

Lernen Sie aus Ihren Fehlern

Das ist nicht so einfach, wie es klingt. Oft werden Sie Ihre Fehler wiedererkennen. 
Übernehmen Sie Verantwortung

Auch für Ihre Misserfolge und die Ihrer Mitarbeiter.

\section{Halten Sie Widersprüche aus}

Die Anforderungen sind oft unvereinbar. Sie können nicht alle erfüllen.

\section{Halten Sie Distanz}

Mitarbeiter, Kollegen und Vorgesetzte sind Mitarbeiter, Kollegen und Vorgesetzte, nicht Freunde. Als Führer sind Sie dementsprechend oft einsam. Diesen Preis müssen Sie bezahlen.

\section{Bauen Sie ein eigenes Netz}

Mit anderen Führungspersonen, aus der eigenen und fremden Organisationen. Alle haben die gleichen Probleme. Ihr Netz ist Ihre Balintgruppe.

Holen Sie Konflikte an die Oberfläche, und tragen Sie sie mündlich aus

Nichtangesprochene Konflikte sind ein Übel. Sie fügen Ihrer Organisation grossen Schaden zu. Nur in ganz ernsten Fällen ist Schriftlichkeit angezeigt.

\section{Beobachten Sie Ihr Umfeld genau}

Auch dann wissen Sie nicht genug.

Nutzen Sie die Energie von Gegenkräften Energie kann umgelenkt und für Sie nutzbar werden.

\section{Erkämpfen Sie sich Spielraum}

Er wird sowieso wieder eingeengt, meist aber nur teilweise.

Halten Sie das Heft des Handelns in Ihren Händen Besetzen Sie wichtige Themen als erster.

Kämpfen Sie auch in der Defensive Verteidigung kann die überlegene Kampfform sein [1].

\section{Verlangen Sie den mündlichen Vortrag}

1 Carl von Clausewitz (1780-1831) Vom Kriege. nur, was Ihre Aufgabe betrifft, besonders in Berichten und Protokollen.

Hören Sie zu

Ganz besonders, wenn Ihnen die Botschaft nicht passt.

Fassen Sie sich kurz, und verlangen Sie dasselbe von Ihrem Team

Mündlich und schriftlich! Eine Seite genügt häufig. Der Rest gehört in die Beilagen.

Wenden Sie das 85/15-Prinzip an

Erst in der Ausführung ist 100prozentige Genauigkeit angezeigt.

Entscheiden Sie, was Sie liegenlassen wollen Sie können gar nicht alle Ihre Aufgaben erfüllen. Es ist erstaunlich, wieviel sich von selbst erledigt.

Gesetze und Vorschriften stehen im Dienst der Aufgabe, nicht der Ausrede

Es gibt immer einen Interpretationsspielraum. Jeder Rechtsanwalt kann Ihnen das bestätigen.

\section{Bereiten Sie Sitzungen vor}

Auch Unterredungen. Dazu gehört gründliches Aktenstudium.

Begreifen Sie Reklamationen als Chance Reklamationen helfen Ihnen, Schwächen zu erkennen.

Verfassen Sie wichtige Protokolle selbst Oder korrigieren Sie von anderen verfasste Protokolle vor der Freigabe.

Entscheiden Sie, ob Sie diktieren oder schreiben Schlechte Schreiber gewinnen viel Zeit mit dem Diktat (auch E-Mails).

Und dann noch: Lernen Sie verlieren Und Misserfolge ohne Leiden wegzustecken.

Und zum Schluss: Freuen Sie sich am Erfolg, und feiern Sie ihn Mit Ihrem Partner und Ihrem Team. 